\title{
Perceive to learn to perceive: an experience with virtual reality devices for architecture design learning
}

\section{SIGRADI2018 TECHNOPOLITICAS \\ xxii congresso da sociedade iberoamericana de gráfica digital 22th conference of the iberoamerican society of digital graphics 07|08|09|novembro|2018 iau usp | são carlos | sp br}

\author{
Guilherme Nunes de Vasconcelos \\ UFMG | Brazil | guiazul@ufmg.br \\ Mateus de Sousa Van Stralen \\ UFMG | Brazil | mateus-stralen@ufmg.br \\ Alexandre Menezes \\ UFMG | Brazil | alexandremmenezes@gmail.com \\ Fernando Murilo Gontijo Ramos \\ UFMG | Brazil | fmgramos2@gmail.com
}

\begin{abstract}
This work investigates the potential use of low-cost virtual reality (VR) devices in architectural education to improve spatial perception of undergraduate architecture students. The experiment involved a gradual approach into the design process, starting with an intervention on a physical space, its bidimensional representation, $3 \mathrm{~d}$ modelling and immersion in VR. After the immersion, students answered a questionnaire with open and closed-questions about their experience, and their evaluation of the use of VR in the designing. The findings point to the use of VR as a means to explore, perceive and reflect on decisions, allowing students a better understanding of designing.
\end{abstract}

Keywords: Virtual reality; Architectural design; Architecture teaching; Representation; Low-cost devices.

\section{INTRODUCTION}

The actual interest in virtual reality (VR) cannot be considered a new concern. Since the 1990s, when the first wave of VR emerged, we saw other two moments of renewed interest in VR technologies, one at the turn of the 21st century and more recently, a third wave took place since 2015 when several companies announced their interest in producing VR devices (Heim, 2017).

In 1965 when Ivan Sutherland published "The Ultimate Display" paper, describing the possibilities of a room in which matter could be controlled by a computer, and any object within will be good enough to be used, the expectations with immersion devices started to burst. In 1968, Sutherland and his student Bob Sproull presented the "Sword of Damocles", a head-mounted display (HMD) which could immerse the user in an environment populated with wireframe $3 d$ models that overlap the physical environment and react to the user head position, paving the way to VR as we know it (Sutherland, 1965).

The "Sword of Damocles" wasn't the first digital HMD. In 1945 Henry J. de McCollum patented the "Stereoscopic Television Apparatus", an object intended for television viewing in a stereoscopic way using cathode ray tubes (McCollum, 1945), which was improved by Morton Heilig in 1960 with "Telesphere Mask". Heilig's Mask incorporated a pair of ear phones, for sound effects and a pair of air nozzles, capable of blowing air in user's face, augmenting the immersion (Heilig, 1960).
A non-HMD immersion device of great importance was presented in 1962 by Heilig, the "Sensorama", a simulator apparatus equipped with image projection, breeze and odor blower, binaural sound, and vibration motors, working in a coordinated way to provide a "new and improved apparatus to develop realism in a simulated situation" (Heilig, 1960, p.1).

The main difference between these devices and Sutherland and Sproull's HMD is the ability to include a certain degree of interactivity with the user through headtracking, placing him "inside a computer-created world, instead of peering in at it through a narrow window" (Rheingold, 1991, p.38).

Although there's a certain dispute over the first time the term "virtual reality" appeared, it was in 1989 when it spread widely through the mouth of VR pioneer Jaron Lanier. As he puts it, virtual reality "recreates our relationship with the physical world in a new plane, no more, no less" and it "only has to do with what your sense organs perceive" (Kelly et al, 1989). Michael R. Heim (1993), a virtual reality theoretician, formulates his definition of VR from merging the dicitionary description of the words "virtual" and reality": "Virtual reality is an event or entity that is real in effect but not in fact" (p.108).

Thenceforth, the VR technology has greatly improved in terms of variety, availability, display resolution, processing power, sensors accuracy and became more affordable. 
For Steinicke (2016) the most important recent development in VR was the association of smartphones with headsets, enabling the experience of VR to be portable, lightweight, and financially accessible. The ubiquity of smartphones freshened and opened up the possibilities to explore VR applications in different fields in an unprecedented way.

In the architecture field there are a wide array of immersive experiences using these low-cost VR devices. The user is can visit, albeit limited to some degree, architectural classic buildings through $360^{\circ}$ videos, or to explore the surroundings of a possible building site, to walk cities from above like a digital-Godzilla, or even to walk through $3 \mathrm{~d}$ modeled buildings that existed, will exist or will never exist in physical world, in a "virtual architectural promenade".

The implications and applications of those possibilities of VR use for architecture education are obvious but still require quite an investigation.

The goal of this work is to study the potential of using lowcost virtual reality (VR) devices to improve aspects related to the spatial perception of undergraduate architecture students. This article presents a theoretical discussion related to this topic and an experiment conducted with undergraduate architecture students at the beginning of their second semester in architecture education, dealing with their first contact with a traditional architectural design.

The proposed exercise was a small-scale architectural intervention in two different rooms of our school. The main steps of the exercise were the measurement of spaces, the conception of the proposal in real scale, bidimensional drawing of the proposal, three-dimensional modeling, immersion in VR and the revision of the proposal after VR experience.

The strategy to approach technical representation from a physical space to it's bidimensional and three-dimensional representation was chosen in order to expose the students to the limits of traditional representation and to introduce them to the interpretation of abstract information in different degrees.

We intended to introduce them gradually to the architectural design process, going from the real space to bidimensional technical representation, then to a threedimensional model, to finally immerse them in VR as a process to generate feedback for the revision of their design.

Each one of these phases could be seen as a feedback generator itself, allowing the student to be aware of the consequences of their moves and to propose changes at each step. In this regard, design could not be understood as a linear process, but as a process that takes into appreciation the unintended consequences of each decision when and by making new moves. As Schön (1983) points, design is a reflective conversation with a situation, where designer reflects-in-action from his earlier moves.
This reflection-in-action is also studied by Glanville (2007) under the name of conversation, a process that can be held between designer and others, or between designer and himself using, for example, pen and paper. Conversation is at the core of the design experience and due to its openness and unpredictability, can lead the designer to unanticipated places, which can introduce novelty in the process.

Since reflection is a key aspect of designing, perception is a condition for reflection as it is only possible to reflect on what is perceived.

Perception, as posted by Von Foerster (1989), is an act that is closer to con-ception, than to a passive state such as in re-ception, to which he complements: "the world as we perceive it is our invention" (p.223).

Glanville (2007) affirms that designing follows a particular path. Normally a problem leads to a solution, but that is not the case when designing: the outcome "can be seen as a solution that defines the problem". This is due to the nature of the design problem itself, as the perception of the problem depends upon one's idea for solving it, which leads us to see that problems of this nature can have endless solutions (Rittel and Webber, 1973).

The main characteristics of these kind of problem, called "wicked problems" by Rittel and Webber (1973), are being ill-defined and having no stopping rule. According to Coyne (2005), the "wicked problems" proposition was an antidote to the understanding of the science of design as proposed in 1969 by Herbert Simon in his book "The Sciences of the Artificial" where Simon suggests a rational approach to design, transforming it into a teachable doctrine.

Glanville (2006) sees virtual reality, in its widest (and wildest) sense, as perhaps an "opportunity to expand their conceptualizing beyond their conventional view of reality". $\mathrm{He}$ also states that "Virtual Reality only becomes interesting and its power is only just acknowledged and beginning to be explored when we let it take us into realities that are not bent to make concessions to the consensus of the familiar reality we like to assert we inhabit" and completes that there is "clearly a message here for architects!” (Glanville (1995).

For the exercise presented in this article, the initial problem - and therefore the solution - was defined beforehand (to collectively design a space). Nevertheless, we suppose VR can help the students to perceive and reflect better on the other problems that will arise when the students start designing.

Earlier studies conducted relating immersion and architecture have shown the potential of this technology in improving spatial perception (Moural et al, 2013; Baltazar et al, 2014; Kreutzberg, 2014, 2015; Dokonal et al, 2015), or as a communication and validation tool (Heydarian et al, 2014), or even studying how to include VR in the design process (Dokonal et al, 2016).

However, among the evaluated studies, qualitative investigation on the relation between spatial perception, bidimensional and three-dimensional representation, and 
immersion in VR were not found in an integrated way, which we intended to address with this work.

\section{METHODOLOGY}

The development of this experiment was based on our former experiences from previous semesters as teachers of this course when it was possible to identify the mishaps faced by students in perceiving three-dimensional aspects of bidimensional representation.

In renovation projects like the one proposed, students usually start designing by using bidimensional sketches and plans to resolve functional questions, such as sectorization of spaces, circulation flows, equipment layout etc. The spatial implications of their design decisions in that phase will only become evident for most students when they model their solutions in threedimensions. It is expected that at this stage the students proceed to reevaluate their solutions and return to the sketches and plans to refine or to develop new solutions, which does not always happen.

The ability to infer about a given spatiality from its twodimensional representation is not trivial and must be constantly constructed and refined. Any tool or strategy that can assist the student in this ability construction should be used to engage them and to reduce the frustration that inevitably troubles those who presents the greatest difficulties in spatial visualization.

In this sense, the recent developments in VR and its increasing price reduction make this technology worth investigating. The experiment proposed intended to observe qualitatively if VR could be used by students as a tool to help them in the construction of an enhanced spatial perception ability and the repercussions of this improved perception on their proposals.

\section{A SMALL-SCALE DESIGN EXERCISE}

The experiment consisted of design and modeling exercises divided into three steps. At the end of those steps, students were asked to answer a questionnaire.

For the first step of the experiment, students were invited to intervene in an existing space in which they had to design a small office collectivelly. They started by measuring and making sketches, plans and sections of the existing space. The objective of this part of the exercise was to make a first approximation of the students with the technical drawing language, although in a simplified form. After taking the first measures and debating about the design of their collective office, the next step was to make a real scale (1:1) sketch of the design using adhesive tape and measure tape. With the adhesive tape, they sketched on the floor and existing walls the elements proposed by them, such as doors, partition walls, furniture, openings, etc.

After finishing the real scale sketch they were asked to take the exact measures of the existing space combined with their sketches and produce individually a $3 d$ model of that space using Trimble SketchUp. The model was then exported to Kubity (2018), a visualization software available for mobile (Android/iOS) and desktop (Windows/OSX), with the ability to allow immersive experiences in virtual and augmented reality without obstructing or significantly increasing students work.

At this phase, each student was free to propose modifications in the collective space, making each proposal more or less unique. After finishing the model each student had to hand draw plans and sections of their design.

Although it may seem counter-intuitive to go from real scale sketches to $3 \mathrm{~d}$ modelling and then back to handdrawing technical representation, when the plans and sections could be extracted from the $3 d$ model itself, we chose to adopt this hybrid strategy to present students the reasoning behind the construction of technical drawings. This strategy is similar to the "hybrid design approach" described by Zuo et al (2010), which combines the use of digital and non-digital tools to improve learning in designing.

The immersion was made using one mid-end and one high-end Android smartphones inserted in two different cases, a Xiaomi Mi VR Play 2 ( US\$20) and a Samsung GearVR ( US\$100). Those two models of cases were used as authors already had them.

After the immersion, the students answered a questionnaire with open and closed-ended questions. This questionnaire was answered by 48 students and the questions inquired about their previous knowledge of technical drawing, their perception about the designed space after the immersion in VR, their evaluation of the use of VR in the design process, and their perception about the three stages of the exercise.

The questionnaire was composed of 12 questions, being 10 closed-ended questions and 2 open-ended. The questions can be categorized into three groups. The first group dealt with their previous experiences and knowledge of technical representation. The second was related to their experience with the VR immersion. The last one was designed to understand how they relate to the different steps of the experiment with their perception of space, scale, and architectural representation.

\section{RESULTS}

In the first group $68,8 \%$ of the students where positive on having attended other courses that used technical representation. With regard to their knowledge about technical representation, on a scale of 1 (insufficient) to 5 (excellent), the answers were $4,2 \%$ with grade 1, 4 or 5 , $33,3 \%$ with grade 2 and $54,2 \%$ graded 3 .

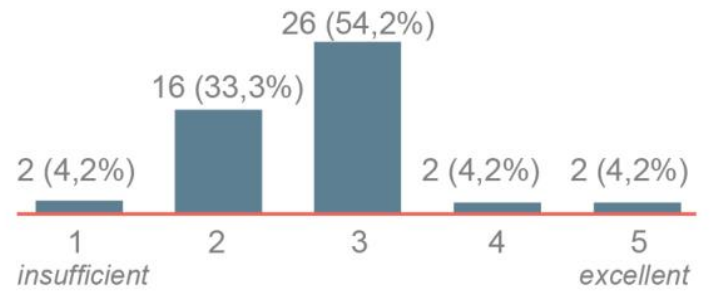

Figure 1: How do you evaluate your knowledge on technical representation until now? (Source: Authors). 
In the second group, the first question inquired about their perception of the designed space after the immersion in VR. 20,8\% classified their space as "Exactly as imagined", $18,8 \%$ as "Different from imagined", 60,4\% "Lightly different from imagined" and none answered "Very different from imagined".

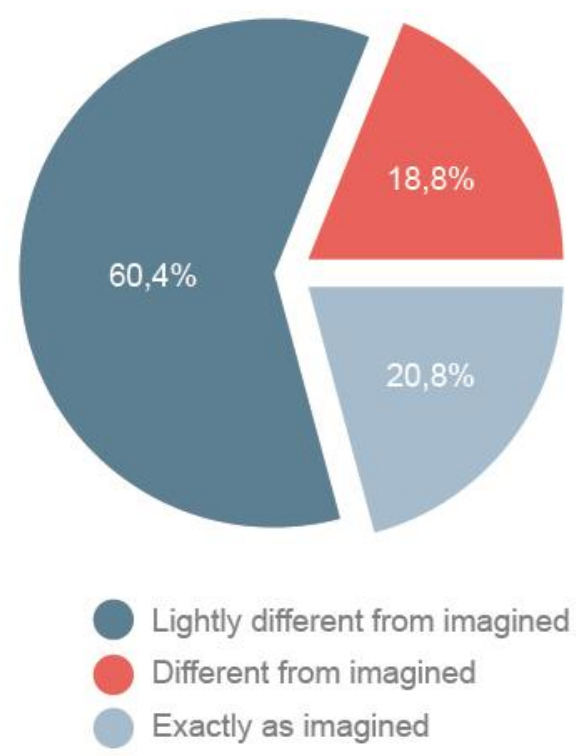

Figure 2: After the immersion in virtual reality, do you think the space is... (Source: Authors).

The next question was if the use of VR enabled them to perceive aspects of their design that was not perceived before and $93,8 \%$ of the answers were positive. The following question was open-ended and about what difference was different perceived via VR about their designs. The answers dealt with their perception about dimensions of elements (furniture, equipment and other objects), dimensions of the space (ceiling height, window height, circulation spaces, etc.) and qualities of the space (space was bigger or smaller than imagined).

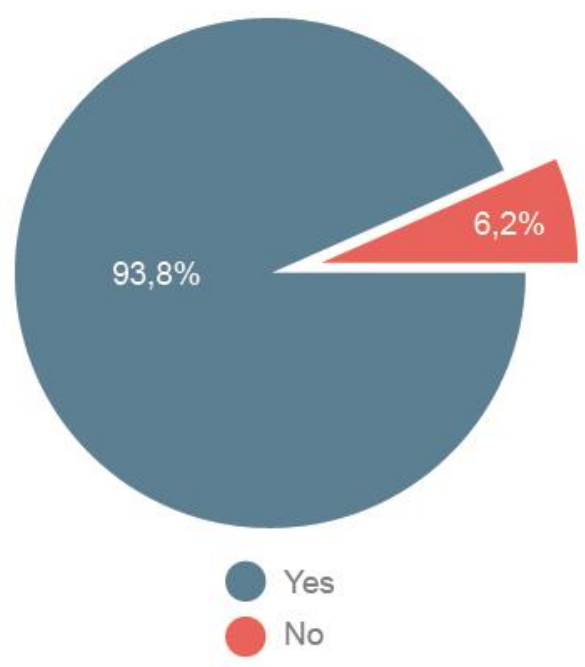

Figure 3: The immersion in virtual reality allowed you to perceive aspects of your Project that wasn't perceived before? (Source: Authors).

One of the most surprising results were related to the question: "After visualizing your project in VR, will you change your proposal?", varying between "No, I'm going to keep it as it is" graded as 1 and "Yes, I'm going to change the proposal radically" graded as 5 . 10,4\% answered 1, 41,7\% ranked themselves $2,22,9 \%$ as 3 , $20,8 \%$ as 4 and $4,2 \%$ as 5 . It's important to point out that the majority $(52,1 \%)$ answered 1 or 2 , signaling that none or little changes would be made after the immersion.

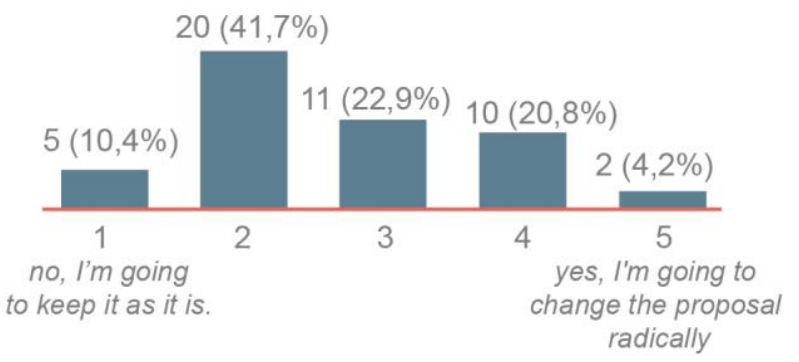

Figure 4: After visualizing your project in VR, you are going to change your proposal? (Source: Authors).

The last question of the second group was open-ended and dealt with how they see the potential of using VR in architecture. The answers were unanimous in recognizing the potential of VR immersion for design and evaluation of the represented space. They were mainly related with the use of VR to improve the communication with clients, or its use as a creative tool assisting in the development of their ideas, and using VR to perceive possible problems and test solutions.

The third group of questions, about the exercise itself, started by asking them if they considered the step of designing in real scale important for their spatial comprehension and for the quality of the final design, measured in a scale from 1 (inutile) to 5 (useful). $10,4 \%$ of the students ranked 3, 18,8\% classified it as 4 and $70,7 \%$ as 5 .

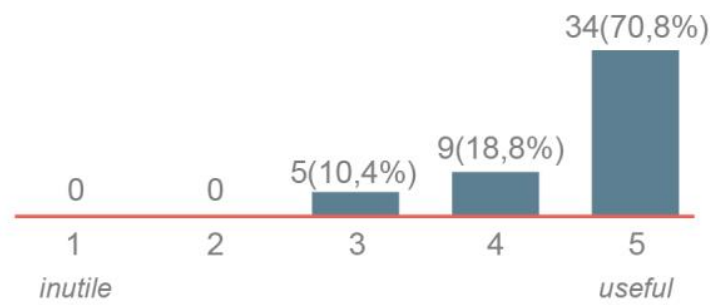

Figure 5: About the first stage of the exercise, designing in 1:1 scale, do you consider this step somehow important for spatial comprehension and for the results obtained in the project?

(Source: Authors).

With regard to the second step of the exercise, when the students had to drawn in real scale their designs made on the first step, they were asked about the level of difficulty for transposing their ideas to the technical representation language, ranging from "very easy" to "very hard" in three distinct aspects: drawing in scale, representation of the elements in the project, and conception of the project (Table 1). 
Table 1: Difficulty level to transpose the proposal to technical drawing language

\begin{tabular}{|c|c|c|c|}
\hline & Draw. in scale & Repres. & Conception \\
\hline V. Easy & $12,5 \%$ & 0 & $2,08 \%$ \\
\hline Easy & $39,5 \%$ & $27,08 \%$ & $14,58 \%$ \\
\hline Medium & $33,3 \%$ & $50 \%$ & $43,75 \%$ \\
\hline Hard & $6,25 \%$ & $22,91 \%$ & $33,33 \%$ \\
\hline V. Hard & $8,33 \%$ & 0 & $8,33 \%$ \\
\hline
\end{tabular}

It is possible to identify in Table 1 that the aspect of "drawing in scale" is considered predominantly easy. The "element representation" aspect is considered of medium difficulty and "conception" is considered mainly between medium and hard.

\section{DISCUSSION}

It has been some time since the field of architecture is trying to benefit from immersive devices to improve or optimize the design process. Dorta et al (1998) already pointed out, 20 years ago, that VR could be of very good use to communicate 3D information in the design process but it should not be seen as a cure-all for design.

As Lanier points out, architecture is one of the strongest precedents in the development of VR, but the possibility of a revolution in the design process due to the use of VR devices seems very unlikely, even though there are significant improvements, especially with respect to the educational use of this technology (Kelly et al, 1989).

According to Glanville (1995), if we keep insisting on using VR as an attempt to just re-create our realities we could lose its potential power. If there is any chance for the so expected revolution through VR it seems to depend on transforming our relation with it, using VR not just for presenting our ideas, but as a medium for testing, exploring, perceiving and, most of all, inventing.

The perception of most students that VR helped to recognize aspects that had not been observed until then, points in this direction and corroborate with the findings of previous studies (Moural et al, 2013; Baltazar et al, 2014; Kreutzberg, 2014, 2015; Dokonal et al, 2015), evidencing once more the potential of VR for the improvement of spatial perception.

There was an expectation that this "improved perception" would generate significant repercussions in the students' proposals, which did not happen. There were several adjustments in dimensions, heights of elements and equipment position, but no radical modifications were identified in the proposals after the immersion in VR.

Dorta et al consider VR the ideal vehicle for generating feedback (Dorta et al, 1998), and we intend to investigate this in more depth on further experiments, but this experiment did not lead students to review their projects beyond mere adjustments. This can possibly be credited to the low complexity of the exercise, since the spatial limits of the existing room were predefined, leaving the students to work mainly with the layout and furniture design. Therefore, it seems necessary to investigate whether the constraints of this exercise were too rigid and limited the students proposals beyond due.
On the other hand, although not presented here, the students demanded in other moments of the course the use of the VR to evaluate and validate some design choices, what seems an indication the desire to include VR as a tool in their design process.

The main limitation in the use of low-end devices at the current state of the technology refers to capturing the variation of the height of the user. It manages to capture head rotation with satisfactory precision, but the height of the user is somehow fixed, which makes it difficult to test issues related to observer height and view.

With regard to the software Kubity, a limitation found was that in the used version it was not possible to share what the user wearing the HMD is seeing in a computer display or another device. This hinders possible conversations among students creating difficulties for them to help each other. It is believed that in future versions of the software this limitation will be overcome.

In the same direction, it will be very useful to have instructors and students immersed in the same virtual environment (which is still not possible today using lowend VR devices) in order to discuss about a design while being inside it. This would certainly change some of the dynamics of architectural design teaching since those "virtual promenades" through their design could reveal as observed in the exercise - unexpected aspects of the project, which would hardly be perceived by traditional representation means.

In relation to the design of the exercise, the strategy of progressively introducing them to technical representation seemed effective. The difficulty encountered by the students to represent the two-dimensional elements was somehow expected and can be considered normal, since it is their first experience with traditional technical representation.

One of the possible strategies in order to better integrate VR in the process is reorganizing the steps of the exercise to enable them to immerse earlier in the model to increase the possibilities for reformulation of their designs, leaving as a last step the preparation of technical drawings.

As we attempted to show with this experiment, there is a possibility of achieving significant gains regarding spatial perception using VR, but there are subtle aspects in the use of this technology that must be taken into account to make its use more interesting and fruitful, going beyond simple technological juggling.

The use of VR for visualization of designs is pertinent, but in addition to perceiving spatial qualities it is desirable that students reflect about what they perceive and re-design their proposals. For this to be possible, the immersion in VR should ideally happen at earlier stages of the design process, not in a specific moment, but continuously, at each time a decision needs to be evaluated and reevaluated.
5 


\section{REFERENCES}

Baltazar, A. P., Souza, E. D. M., Pontes, M. M., Gonçalves, F. S., \& Metzker, L. S. (2014). Ambiente de imersão virtual como ferramenta para mudança de paradigma no processo de projeto arquitetônico: da representação à interação. Blucher Design Proceedings, 1(8), 551-555.

Coyne, R. (2005). Wicked problems revisited. Design Studies, 26(1), 5-17.

Dokonal, W., Knight, M. W., \& Dengg, E. A. (2015). New Interfaces-Old Models. In Real Time-Proceedings of the 33rd eCAADe Conference (Vol. 1, pp. 101-106)

Dorta, T. (1998). The Impact of Virtual Reality on the Design Process. In Digital Design Studios: Do Computers Make a Difference in Design Studio (p. 138-160).

Glanville, R. (1995). Architecture and Computing: a medium approach. Computing in Design: enabling, Capturing and Sharing Ideas-ACADIA, 95.

Glanville, R. (2006). Construction and design. Constructivist Foundations, 1(3), 103-110.

Glanville, R. (2007). Try again. Fail again. Fail better: the cybernetics in design and the design in cybernetics. Kybernetes, 36(9/10), 1173-1206.

Heilig, Morton L. (1961). US Patent No. US3050870A. New York, USA: United States Patent Office.

Heim, M. (1993). The metaphysics of virtual reality. New York: Oxford Univ. Press.

Heim, Michael R. (2017). Virtual Reality Wave 3. In Gackenbach, J., \& Bown, J. (Orgs.). Boundaries of self and reality online: implications of digitally constructed realities. (pp.261-277). London: Academic Press, an imprint of Elsevier.

Heydarian, A., Carneiro, J. P., Gerber, D., Becerik-Gerber, B., Hayes, T., \& Wood, W. (2014). Immersive virtual environments: experiments on impacting design and human building interaction.
Kelly, K., Heilbrun, A., \& Stacks, B. (1989). Virtual reality: an interview with Jaron Lanier. Whole Earth Review, 64, 108120.

Kreutzberg, A. (2014). New Virtual Reality for Architectural Investigations. In Fusion-Proceedings of the 32nd ECAADe Conference, 1, 253-260.Dokonal et al, 2015

Kreutzberg, A. (2015). Conveying Architectural Form and Space with Virtual Reality. In Real Time-Proceedings of the 33rd ECAADe Conference, 1, 117-124.

Kubity Speak3d. (2018). Retrieved from https://www.kubity.com/.

McCollum, H.J. de N. (1945). US Patent No. US2388170A. Chicago, USA: United States Patent Office.

Moural, A., Eloy, S., Sales Dias, M., \& Pedro, T. (2013). How Space Experimentation Can Inform Design: Immersive Virtual Reality as a Design Tool (p. 182-185). Sigradi 2013.

Rheingold, H. (1991). Virtual Reality. New York, USA: Summit Books.

Rittel, H. W., \& Webber, M. M. (1973). Dilemmas in a general theory of planning. Policy sciences, 4(2), 155-169.

Schon, D. A. (1983). The Reflective Practitioner: How Professionals Think in Action. USA: Basic Books.

Steinicke, F. (2016). Being Really Virtual. Springer.

Sutherland, I. E. (1965). The ultimate display. Multimedia: From Wagner to virtual reality, 506-508.

Von Foerster, H. (1972). Perception of the future and the future of perception. Instructional Science, 1(1), 31-43.

Zuo, Q., \& MaloneBeach, E. E. (2010). A comparison of learning experience, workload, and outcomes in interior design education using a hand or hybrid approach. Family and Consumer Sciences Research Journal, 39(1), 90-106. 